\title{
Simultaneous Reconstruction of Tissue Attenuation and Radioactivity Maps in SPECT
}

\author{
Yi Tian ${ }^{1}$, Huafeng Liu ${ }^{1}$, and Pengcheng Shi ${ }^{2,3}$ \\ ${ }^{1}$ State Key Laboratory of Modern Optical Instrumentation \\ Zhejiang University, Hangzhou, China \\ ${ }^{2}$ School of Biomedical Engineering, Southern Medical University, Guangzhou, China \\ ${ }^{3}$ Department of Electrical and Electronic Engineering \\ Hong Kong University of Science and Technology, Hong Kong \\ \{eeliuhf, eeship\}@ust.hk
}

\begin{abstract}
The importance of accurate attenuation correction in single photon emission computed tomography (SPECT) has been widely recognized. In this paper, we propose a novel scheme of simultaneous reconstruction of the tissue attenuation map and the radioactivity distribution from SPECT emission sinograms, which is obviously beneficial when the transmission data is missing for cost or efficiency reasons. Our strategy combines the SPECT image formation and data measurement models, whereas the attenuation parameters are treated as random variables with known prior statistics. After converting the models to state space representation, the extended Kalman filtering procedures are adopted to linearize the equations and to provide the joint estimates in an approximate optimal sense. Experiments have been performed on synthetic data and real scanning data to illustrate abilities and benefits of the method.
\end{abstract}

\section{Introduction}

Single photon emission computed tomography provides functional information of the physiological processes of interests. Recently, SEPCT has enjoyed increasingly widespread use, because its costs and technology are within the reach of most clinical nuclear medicine facilities. The physical basis of SPECT is to reconstruct the radioisotope labeled tracer distribution externally through collection of photons that are emitted from inside the human body. However, due to the attenuation of the photon during it traveling through various tissues, the image quality and the quantitative accuracy of SPECT reconstructions degrade greatly from the true distribution. Thus, there have been considerable efforts within the SPECT community aimed at incorporating attenuation correction into any given reconstruction method [5].

Earlier approaches of attenuation correction in SPECT largely fall into two groups. The first one attempts to measure the attenuation map based on transmission scanning, including an external radionuclide source [45, CT [7], or segmented MRI data 8. However, because the emitted photon traverses only part of the patient's anatomy before reaching detector, the problem of photon attenuation in SPECT has proven to be very difficult. There is still a need to 
develop appropriate strategies for accurate and reliable attenuation map recovery based on transmission scanning data. The second group attempts to recover the attenuation map without adding a separate transmission scan. Some approaches involve using a known/segmented object contour with a priori distribution of the attenuation coefficient. However, while manual contour delineation is very complex and time consuming, automatic edge-detection remains one of the open issues in the medical image analysis. On the other hand, in practical situations, especially for pathological data sets, it is almost impossible to have the exact patient-dependent attenuation information a priori. In addition to the prior methods, the incorporation of consistency conditions and statistical modeling into simultaneous estimation of activity and attenuation map from emission data has received a large amount of attention [1016]. These iterative reconstruction algorithms require careful modeling of the imaging system response model which is subject to a number of physical effects. How to process the effects of the uncertainties in the model remains an important issue 39 .

In this paper, we present a joint estimation approach to recover the tissue attenuation and radioactivity distributions simultaneously from emission sinograms. Our efforts have two significant novel aspects. First, with the SPECT imaging and measurement processes in state space representation, the attenuation parameters are treated as random variables with known prior statistics in our effort. Thus, the proposed scheme unified the attenuation map reconstruction problem from transmission scan or emission sinogram data into a generalized framework. Secondly, while in general accurate identification of attenuation from emission data is difficult, our approach undertakes the data and model uncertainties naturally by formulating the problem as an extended state estimation effort on the attenuation parameters. In our current implementation, the extended Kalman filter (EKF) procedures are adopted to linearize the augmented state representation and provide joint/simultaneous estimates in the minimummean-square-error sense. An evaluation study on simulated phantom data and real imaging data is described, where experimental results have shown great promise for such strategy.

\section{Methodology}

\subsection{SPECT Scan Modeling}

In SPECT imaging, the emission sinogram data, acquiring by a rotating detector head around the patient at each angel, can be represented lexicographically as a column vector $y=\left\{y_{i} \mid i=1, \ldots, M\right\}$. Here $i$ indicates different projection defined by rotating angel and different detector bin, and $M$ is the total number of projections. During an emission scan, photon will be attenuated by body tissue before reaching the detector along its travelling path. Assuming that the activity distribution and attenuation map are given by column vectors $x=$ $\left\{x_{j} \mid j=1, \ldots, N\right\}$ and $\mu=\left\{\mu_{j} \mid j=1, \ldots, N\right\}$, one photon's survival probability after attenuation is equal to linear integration along its path in the direction 

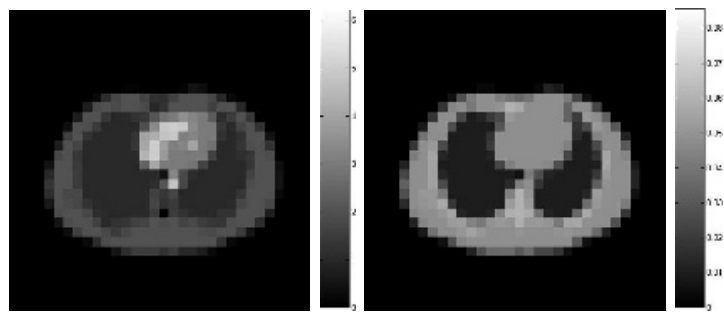

Fig. 1. A digital Zubal phantom: activity distribution (left) and attenuation coefficient distribution (right)

towards the detector. Where $x_{j}$ and $\mu_{j}$ represent the the activity and the linear attenuation coefficient in voxel $j$, and $N$ is the total number of image voxels.

Let $l_{i j k}$ represent the probability that the photon generated in voxel $j$ passing voxel $k$ and attenuated by $\mu_{k}$ along a projection line $i$ when emitted into the detector. The photon survival probability considering about attenuation can be represented by a matrix $\mathrm{A}$ with elements $[A]_{i j}=a_{i j}$ as

$$
a_{i j}=\exp \left(-\sum_{k} l_{i j k} \mu_{k}\right)
$$

If we use $d_{i j}$ to indicate the probability that a photon produced in voxel $j$ reaches the front face of detector along projection $i$ without attenuated, then represent all of these probability with a 3 dimensional vector $[L]_{i j k}=l_{i j k}$ and a system matrix $[D]_{i j}=d_{i j}$, the mathematical model of the SPECT emission measurement could be written as 1

$$
y=(A \cdot D) x+\text { noise }=\left(e^{-[L \mu]} \cdot D\right) x+\text { noise }
$$

or in a discrete form

$$
y_{i}=\sum_{j}\left[\exp \left(-\sum_{k} l_{i j k} \mu_{k}\right) d_{i j} x_{j}\right]+\text { noise }
$$

\subsection{State Space Representation of SPECT Imaging}

In emission tomography such as SPECT, the goal is to reconstruct the emission/radioactivity distribution $x$ from the projected measurement data $y$ (the photon counts). The state equation of the imaging system, which describes the radioactivity of the pixels, can be written in the form of

$$
x(t+1)=S x(t)+v_{s}
$$

with some initial activity $x_{0}$. The system noise $v_{s}$ models the statistical uncertainty of the imaging model. In general, equation (4) represents the dynamic

\footnotetext{
${ }^{1}$ Note that the symbol '' means the dot product of 2 matrixes.
} 

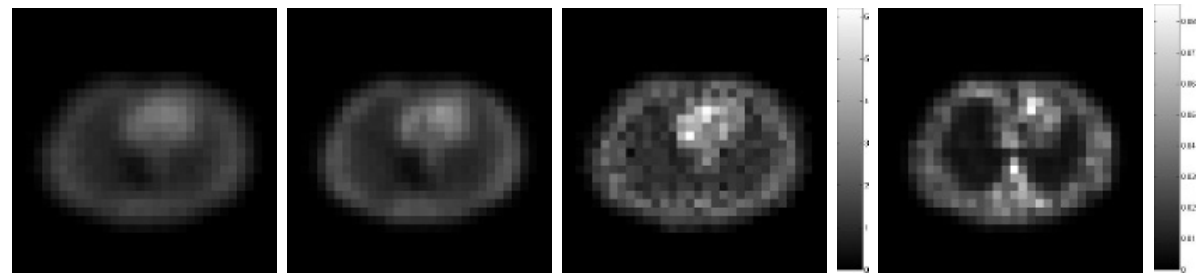

Fig. 2. From left to right: activity maps recovered by FBP, EM-ML, and our method, and the attenuation map estimated by our framework

changes of the state variable $x$, and it reduces to the conventional static reconstruction problem when the transition matrix $S$ is an identity matrix. The associated measurement equation (2), which describes the observations provided by the imaging data $y(t)$, can be expressed in a more general form:

$$
y(t)=G x(t)+e_{o}
$$

where $G$ is a known measurement matrix, and $e_{o}$ is the measurement noise.

\subsection{Augmented State Space Representation of SPECT Imaging}

Equations (4) and (5) provide a so called state-space representation of the SPECT system with assumed known attenuation coefficients. In practice, however, the attenuation data are not precisely known. In this work, with the attenuation coefficients treated as random variables with known or assumed prior distributions, we are trying to determine the best estimates of the spatial distributions of the activity and attenuation map simultaneously.

In order to perform the joint estimation, the unknown state vector $x$ is augmented by the unknown attenuation coefficient vector $\mu$ to form the new state vector $z=\left[\begin{array}{ll}x & \mu\end{array}\right]^{T}$. Since $\mu$ is a temporally constant, spatially varying parameter and treat $x$ as static for one frame of the image, equation (4) is converted to the new augmented state equation:

$$
z(t+1)=z(t)+v(t)
$$

Here $v$ represents the uncertainties of the model, with $E[v(m) v(n)]=Q(t) \delta_{m n}$.

In a similar fashion, the new augmented measurement equation is derived from equation (2) and (5):

$$
y(t)=f(z(t))+e(t)
$$

with

$$
\begin{array}{r}
f(z(t))=\left(e^{-[L \mu(t)]} \cdot D\right) x(t) \\
E[e(t)]=0, E\left[e(m) e(n)^{\prime}\right]=R(t) \delta_{m n}
\end{array}
$$

where $e$ represents the uncertainties of the measurement. By solving $\hat{z}(t)$ from above nonlinear state space equation, the estimation of activity map $\hat{x}(t)$ and attenuation map $\hat{\mu}(t)$ can be obtained simultaneously. 

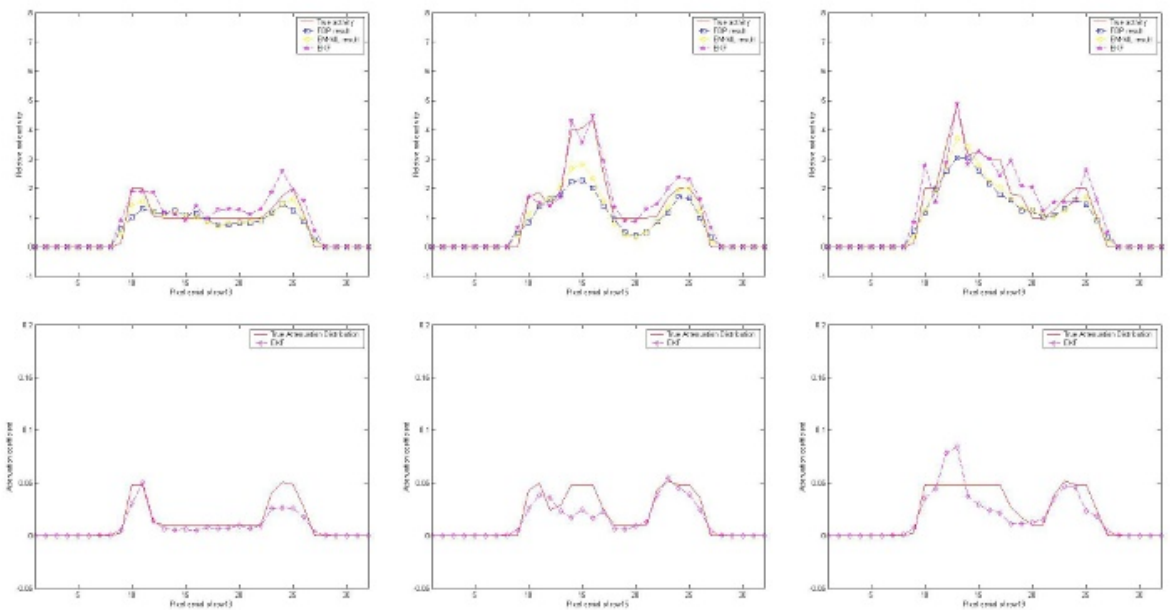

Fig. 3. Top: Vertical profiles along $13^{\text {th }}$ column (left), $15^{\text {th }}$ column (middle), $19^{\text {th }}$ column (right) of recovered activity maps. Bottom: Vertical profiles along $13^{\text {th }}$ column (left), $15^{\text {th }}$ column (middle), $19^{\text {th }}$ column (right) of recovered attenuation maps.

It is known that the noises in emission sinogram is Poisson distributed. However, by applying the Anscombe transformation, the Poisson noise could be converted into Gaussian one [2]. If $x$ is Poisson distributed with mean equal to $\bar{x}$, then $(x+3 / 8)^{1 / 2}$ can be approximated as Gaussian distributed with its mean equal to $\bar{x}+1 / 8$ and its variance approximately equal to 0.25 [2]. Thus this form of state space formulation (equations (6) and (7)) leads to a solution of the filtering problem using the extended Kalman filter (EKF).

\subsection{Joint Estimation of Tissue Attenuation and Radioactivity Maps: An EKF Solution}

To solve the nonlinear state space equations (6)-(7) with a measurement description (8), we adopt an EKF approach to estimate the state variables. In order to apply the EKF, the nonlinear function $f$ is recursively linearized around the most recent estimate of $z(t)$. By taking gradient of the transformation function at time $\mathrm{t}$ according to $H(t)=\left.\frac{\partial f(z)}{\partial z^{T}}\right|_{z=\hat{z}^{-}(t)}$, the estimated value $\hat{z}(t)$ of vector $z(t)$ can be carried out by the following prediction-correction iterative process.

\section{Prediction}

$$
\begin{aligned}
\hat{z}^{-}(t+1) & =\hat{z}(t) \\
P^{-}(t+1) & =P(t)+Q
\end{aligned}
$$

\section{Correction}

$$
\begin{aligned}
\hat{z}(t) & =z^{-}(t)+K(t)\left[y(t)-f\left(\hat{z}^{-}(t)\right)\right] \\
P(t) & =[I-K(t) H(t)] P^{-}(t) \\
K(t) & =P^{-}(t) H^{T}(t)\left[H(t) P^{-}(t) H^{T}(t)+R\right]^{-1}
\end{aligned}
$$


Here, the Kalman gain matrix $K(t)$ drives the improvement of $z \hat{(t)}$ and $P$ in the correction step by weighting the new information coming from the next system measurement $y(t)$ and the previously stored information in the prediction step. The initialization at time $t=0$ of the state vector $z(0)$ and the relevant covariance matrix $P(0)=E\left\{[z(0)-\hat{z}(0)][z(0)-\hat{z}(0)]^{T}\right\}$ are given at the beginning of the iteration. The covariance of the measurement error $R$ and system error $Q$ is assumed to be known and set to time-invariant.

\section{Experiments and Discussions}

\subsection{Digital Phantom Study}

A digital Zubal thorax phantom with known radioactivity concentrations and attenuation coefficient distribution is used, as shown in Fig 1, to evaluate our method. The resolution of the original image is 32 by 32 pixels, and 1024 projections over 360 degrees are simulated for a rotating SPECT detector head. To generate realistic data, we simulate the single photon emission events considering tissue attenuation and detection probabilities, the measured sinogram $y$ is created based on equation (2), here the noise mainly is 30 percents scatter events.

With this set of synthetic data, three types of reconstruction methods (FBP, EM-ML and our method) are then used. The activity maps obtained by our method and other methods are shown in Fig,2, and the attenuation map reconstructed from the emission data by our simultaneous estimation is also shown in the same figure. The EM-ML and FBP method with unknown attenuation map settings produce some noticeable errors. The proposed joint estimation framework gives the best estimates. From the vertical profiles, as shown in Fig. 3, we can draw the same conclusion more clearly.

\subsection{Real Phantom Scanning Data with a Clinical Modality}

The second data set used in this study was acquired on the SIEMENS ECam ${ }^{\text {duet }}$ SPECT scanner with a real cylinder phantom. The dimension of the phantom is $200 \mathrm{~mm}$ (diameter) x 290mm (depth). A Teflon rod and two two hollow PMMA cylinders with diameters of $50 \mathrm{~mm}$ are inserted in the phantom's volume, as shown in Fig. 4 .

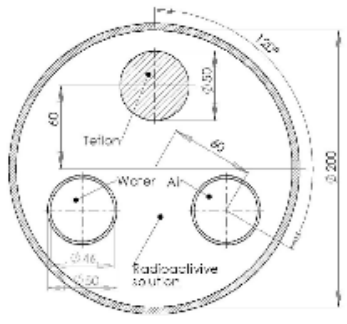

Fig. 4. A physical imaging phantom with 3 different material rods inside 

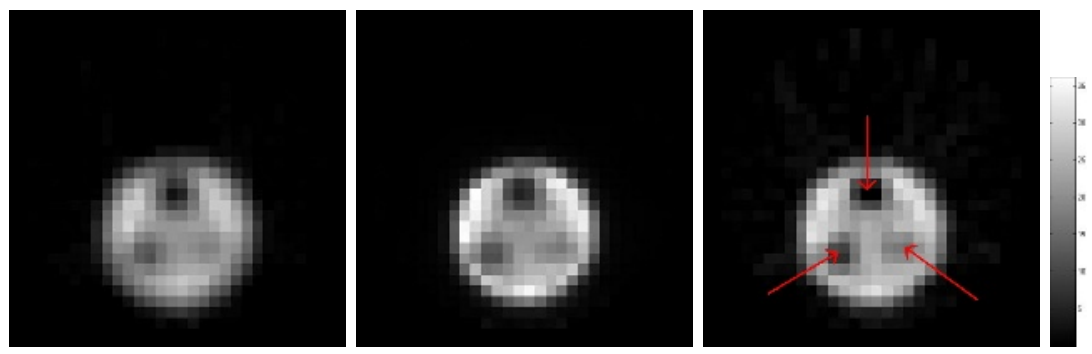

Fig. 5. Reconstructed activity map of real phantom data by FBP (left), EM-ML (middle), and our method (right), where the arrows indicate cold areas

The phantom is filled with ${ }^{99 m} \mathbf{T c}$ concentration with a total radioactivity of $20 \mathrm{mCi}(100 \mathrm{kBq} / \mathrm{cc})$ and the two hollow cylinder rods are filled with air and pure water respectively. The phantom was scanned with a SIEMENS ECam ${ }^{\text {duet }}$ ECT scanner by two detector head rotating at total 64 angle position around 180 degree and the acquiring time at each position is 30 seconds. The final sinogram data has $64 \times 128$ projections for each slice. Then FBP, EM-ML method and our algorithms as described in the previous sections have been applied to recover images from the measured data, as shown in Fig [5] It is obvious that our method produces the best reconstruction results, especially for the three cold areas.

\subsection{Discussions}

Attenuation correction from transmission data is a well-documented research topic. Actually, the attenuation and activity distributions can be simultaneously reconstructed based on our proposed framework if both transmission and emission data are available. In this case, the measurement vector $y$ consists of the emission data $y_{1}$ and transmission scanning measurement $y_{2}: y(t)=\left[\begin{array}{ll}y_{1} & y_{2}\end{array}\right]^{T}$. Thus, the measurement equation of the state space representation is converted into: $y(t)=\left[\begin{array}{cc}G(t) & 0 \\ 0 & A_{\text {tran }} \cdot D\end{array}\right]\left[\begin{array}{c}x(t) \\ x_{\text {tran }}(t)\end{array}\right]+e(t)$. Where the matrix $G$ is the dot product of $A, D$ and $L$ which defined in section 2.1 as $G(t)=A \cdot D=e^{-[L \mu(t)]} \cdot D$, where $x_{\operatorname{tran}}(t)$ is the known effective activity distribution of the external transmission source and the diagonal matrix $A_{\text {tran }}$ is the transmission attenuation factor matrix along every transmission projection, which diagonal element has the form as $a_{i i}^{t r a n}=\exp \left(-\sum_{j} l_{i j} \mu_{j} t\right)$. Here $l_{i j}$ represents the effective intersection length along projection $i$ passing voxel $j$, and $\mu_{j}$ is the attenuation coefficient of voxel $j$. This form of formulation leads to a solution of the filtering problem based on extended Kalman filter algorithm.

As a straightforward extension, based on nonlinear $\mathcal{H}_{\infty}$ filter, we can reconstruct the activity map with taking into account for the attenuation effects. The mini-max $\mathcal{H}_{\infty}$ criterion does not impose restrictions on the noise type, as long as it is of finite energy, and thus is more robust and less sensitive to noise variations and modeling assumptions. Detailed investigations on these issues are underway. 
Acknowledgments. This work is supported by the National Basic Research Program of China (2003CB716100), the Hong Kong Research Grants Council (HKUST6151/03E), the NSF of China for Innovative Research Groups (60021201), and the Open Foundation of State Key Laboratory of Modern Optical Instrumentation at Zhejiang University (LMOI-0403).

\section{References}

1. A.Krol, J.E.Bowsher, S.H.Manglos, D.H.Feiglin, M.P.Tomai, and F.D.Thomas. An em algorithm for estimating spect emission and transmission parameters from emissions data only. IEEE Trans. Med. Imag., 20:218-232, 2001.

2. F.J.Anscombe. The transformation of poisson, binomial and negative-binomial data. Biometrika, 35:246-254, 1948.

3. F.J.Beekman, C.Kamphuisa, M.A.Kingb, P.P.van Rijka, and M.A.Viergevera. Improvement of image resolution and quantitative accuracy in clinical single photon emission computed tomography. Comput. Med. Imaging Graph, 25:105-111, 2001.

4. H.H.Barrett and W.Swindell. Radiological Imaging: The Theory of Image Formation, Detection, and Processing. CA: Academic, San Diego, 1981.

5. H.Zaidi and B.Hasegawa. Determination of the attenuation map in emission tomography. J. Nucl. Med., 44:291-315, 2003.

6. J.Nuyts, P.Dupont, S.Stroobants, R.Benninck, L.Mortelmans, and P.Suetens. Simultaneous maximum a posteriori reconstruction of attenuation and activity distributions from emission sinograms. IEEE Trans. Med. Imag., 18:393-403, 1999.

7. J.S.Fleming. A technique for using ct images in attenuation correction and quantification in spect. Nucl. Med. Commun., 10:83-97, 1989.

8. N.P.Rowell, J.Glaholm, M.A.Flower, B.Cronin, and V.R.McCready. Anatomically derived attenuation coefficients for use in quantitative single photon emission tomography studies of the thorax. Eur. J. Nucl. Med., 19:36-40, 1992.

9. R.M.Lewitt and S.Matej. Overview of methods for image reconstruction from projections in emission computed tomography. Proc. of the IEEE, 91:1588-1611, 2003.

10. Y.Censor, D.Gustafson, A.Lent, and H.Tuy. A new approach to the emission computerized tomography problem: simultaneous calculation of attenuation and activity coefficients. IEEE Trans. Nucl. Sci., 26:2275-2279, 1979. 